\title{
Commercialisation des moutons à Bouaké, Côte d'Ivoire : variations des prix sur le marché de petits ruminants
}

\author{
G. Toure ${ }^{1 *}$ Z. Ouattara ${ }^{1}$ V. Yapy-Gnaore ${ }^{1}$ \\ T. Yo ${ }^{1}$ K.G. Tanoh ${ }^{1}$
}

Mots-clés

Ovin - Commercialisation - Vente au détail - Prix - Côte d'Ivoire.

\begin{abstract}
Résumé
Une enquête à l'aide d'un questionnaire à passage unique a été conduite auprès des clients de moutons sur le marché à bétail de Bouaké. L'objectif a été de déterminer le comportement du prix du mouton au cours de l'année. La collecte des données, qui a accordé une attention particulière aux périodes des grandes fêtes de l'année, a porté sur le prix et quelques caractéristiques physiques des animaux. L'enquête a concerné 552 moutons commercialisés sur le marché. Le nombre de moutons vendus durant la semaine précédant chacune des fêtes a été plus élevé que celui obtenu après chaque fête. Le marché de petits ruminants de Bouaké a surtout été approvisionné par des moutons importés (81 p. 100), en majorité de type Sahélien (60 p. 100). La manière traditionnelle de fixer le prix des moutons par une appréciation visuelle de la corpulence, sans instrument de mesure, a donné des résultats comparables à la détermination du prix à l'aide de pesées. Les vendeurs ont fait montre d'une bonne expérience dans cette activité. Le poids vif et les périodes de fêtes ont influencé significativement le prix du mouton. Les moutons de grande taille, en majorité de type Sahélien, ont surtout été vendus pendant les périodes de fêtes musulmanes. Durant les semaines précédant les fêtes, le prix du mouton le plus élevé a été enregistré pour la Tabaski (en moyenne 39705 Fcfa/mouton Sahélien et 26750 Fcfa/mouton Djallonké) ; le prix du mouton le plus faible a été obtenu pour la Noël pour le mouton Sahélien (28 105 Fcfa/tête) et au nouvel an pour le mouton Djallonké (17 937 Fcfa/tête). Le prix des moutons a surtout été lié au poids des animaux et aux périodes de fêtes de l'année qui ont modulé le choix du client.
\end{abstract}

\section{INTRODUCTION}

En Côte d'Ivoire, les petits ruminants sont intégrés dans le système traditionnel de production agricole. Ils constituent une source de revenus pour les populations (3) et une source de production de viande (1). Ils jouent aussi un rôle très important dans la vie socioéconomique des communautés africaines, à l'occasion des célébrations des cérémonies religieuses, des mariages $(3,7)$, des rites funéraires, de dons et de confiage. En revanche, l'autoconsommation

\footnotetext{
1. Centre national de recherche agronomique (Cnra), station Elevage, 01 BP 633, Bouaké 01, Côte d'Ivoire

* Auteur pour la correspondance

Gnénékita, Cnra/SRT, BP 31, Bingerville, Côte d'Ivoire
}

ordinaire est rare, comme au Cameroun où on n'abat un animal qu'à l'occasion de fête importante ou en l'honneur d'un hôte de marque (18). Une enquête d'opinion a cependant montré que la viande de mouton était la plus prisée par le consommateur ivoirien (3). Comme au Cameroun, la viande de mouton est consommée dans les grandes occasions, généralement sous la forme d'un plat, nommé ndomba, très apprécié par la population (18).

La ville de Bouaké, cosmopolite sur le plan national, représente un carrefour important où vivent différents peuples du pays et d'autres pays de l'Afrique de l'Ouest, avec une forte proportion de musulmans qui sont de grands consommateurs d'ovins à l'occasion de la fête religieuse de la Tabaski (3). Le marché de Bouaké est ravitaillé par des animaux provenant des élevages urbains de la commune, des élevages ruraux et des pays sahéliens (Mali, Burkina Faso et Niger). 
Si les moutons d'origine étrangère sont apportés sur le marché par des commerçants professionnels, la majorité des moutons locaux appartiennent aux éleveurs. Des deux objectifs principaux de cette étude, le premier tentera d'apporter des éléments de réponse à la question: à quel moment est-il avantageux pour l'éleveur de commercialiser les animaux sur ce marché ? Par ailleurs, la transaction relative aux animaux sur le marché est pratiquée par simple observation de la morphologie de l'animal et le prix est fixé par tête. Ce prix subit des variations diverses, comme au Sénégal où le choix du mouton pour la Tabaski est guidé par des préférences religieuses associées à des critères d'origine économique (12). Le second objectif tentera de répondre à la question: dans cette méthode de fixation des prix, le poids vif estil déterminant du prix de l'animal, comme dans la méthode de fixation de prix par pesée de l'animal, couramment utilisée sur les marchés modernes ? Pour répondre à ces deux questions, durant un an, le comportement du prix de mouton sur le marché de petits ruminants de Bouaké a été suivi afin de déterminer ses facteurs de variation.

\section{MATERIEL ET METHODES}

\section{Caractéristiques du marché}

L'enquête a été menée sur le marché des petits ruminants de Bouaké qui est un espace ouvert, accessible par plusieurs endroits. Les moutons sur lesquels a porté l'enquête étaient détenus soit par des vendeurs intermédiaires qui représentaient les propriétaires absents sur le marché, soit par des vendeurs propriétaires eux-mêmes. Les interlocuteurs des clients étaient représentés par les propriétaires et les intermédiaires qui ne pouvaient être distingués que s'ils précisaient leur rôle lorsque cela leur était demandé. Les intermédiaires recevaient en confiage les moutons qu'ils se chargeaient de commercialiser. Les animaux séjournaient sur le marché et étaient nourris avec des fourrages et des aliments complémentaires achetés sur place. Les clients étaient surtout des particuliers et rarement des bouchers, des rôtisseurs de viande et des éleveurs. Les mouvements d'animaux sur le marché étaient complexes. En effet, après deux mois de présence sur le marché, les commerçants déplaçaient leurs animaux vers d'autres marchés du pays. Les éleveurs vendeurs ramenaient leurs moutons dans les élevages au bout d'un à sept jours de séjour au marché en général. Chaque jour des animaux pouvaient ainsi arriver sur le marché et d'autres en repartir.

\section{Détermination du prix des animaux}

La détermination du prix de l'animal en vente était basée sur une appréciation visuelle de la conformation et sur des critères objectifs relatifs au phénotype de l'animal (couleur de la robe, présence ou absence des cornes, longueur du cou, de la queue, taille des testicules pour le mâle) dont certains sont recherchés par le client. A ces facteurs liés à l'animal, s'ajoutaient certains évènements sociaux (cérémonies religieuses ou festives) augmentant la demande, ce que le vendeur prenait en compte. Par ailleurs, indépendamment de la période des transactions commerciales, le prix de l'animal était plus élevé lorsque le client indiquait imprudemment que l'animal était destiné à un rite traditionnel (sacrifices, funérailles).

\section{Collecte de données}

L'enquête, d'une durée d'un an (de juillet 2001 à juin 2002), a été conduite à l'aide d'un questionnaire fermé et d'entretiens. Le questionnaire a porté sur les animaux vendus. Les moutons vendus non enquêtés par refus du client ont été simplement comptés. L'un des enquêteurs a assisté aux différentes transactions entre le vendeur et le client à l'intérieur du marché. Les trois autres, placés à la grande sortie du marché afin de pouvoir accéder au maximum de clients, ont fait les mensurations sur des animaux issus des transactions.
Les données recueillies sur ces animaux ont été quantitatives et qualitatives. Les données quantitatives ont été relatives au poids et à l'âge de l'animal, à la mesure du périmètre thoracique, de la hauteur au garrot, aux longueurs de la queue, de l'oreille, des cornes et du cou.

Le poids vif des animaux a été obtenu à l'aide d'un peson dynamométrique. Un ruban zootechnique a été utilisé pour la mesure du périmètre thoracique, de la hauteur au garrot et la longueur des cornes, de l'oreille et de la queue. L'âge des animaux a été déterminé à l'aide d'une table dentaire (11).

Les données qualitatives ont porté notamment sur le type, la couleur de la robe et le pays d'origine des animaux vendus sur le marché à bétail. Les deux premières variables ont été déterminées par observation et la dernière par entretien avec le vendeur. Le prix de vente de l'animal correspondait à la somme versée par l'acheteur.

Le rythme de collecte des données a été variable. Dans le premier cas, en période de fêtes, la collecte se faisait quotidiennement durant 14 jours centrés sur les fêtes. Seules quatre fêtes ont été retenues pour cette étude : les fêtes de Noël et du nouvel an, de Pâques, de la Tabaski et de fin Ramadan. Le couple de fêtes Noël et nouvel an a été désigné par Noël dans la suite de l'exposé. Par souci de commodité, l'enquête n'a pas eu lieu les jours fériés, les samedis et les dimanches, quelle qu'ait été leur position par rapport au jour de la fête.

Dans le second cas, en dehors des périodes de fêtes, la collecte se faisait deux fois par mois, à savoir le premier mardi et le dernier vendredi de chaque mois, ceci afin de minimiser la différence du pouvoir d'achat des clients qui était plus élevé au début qu'en fin de mois, surtout pour les salariés.

\section{Traitement des données}

La longueur des cornes a été définie comme un facteur à trois classes : mouton à petite corne (longueur de la corne $\leq 13,5 \mathrm{~cm}$ ), mouton à moyenne corne $(13,5 \mathrm{~cm}<$ longueur de la corne $\leq 17,5 \mathrm{~cm})$ et mouton à longue corne (longueur de la corne $>17,5 \mathrm{~cm}$ ). Les paramètres morphologiques des animaux et leur prix de vente ont été soumis à une analyse de composante principale (ACP) normée afin de déterminer leurs niveau de relations. L'ACP a été réalisée avec sept variables : prix de l'animal entier, poids vif, hauteur au garrot, périmètre thoracique, longueur de la queue, longueur des cornes, longueur des oreilles de l'animal.

Une analyse par régression linéaire de variables quantitatives et qualitatives sur le prix par mouton a été faite à l'aide du modèle linéaire généralisé (type III) pour révéler l'influence éventuelle de ces facteurs, selon le modèle : prix $=A+B_{i} X_{i}+E_{i}$ où $X_{i}$ représente les variables poids vif, hauteur au garrot, périmètre thoracique, longueur de la queue, longueur des cornes, longueur des oreilles, couleur de la robe, race, pays d'origine de l'animal, semaine de collecte. La période de fête a été codée en semaine avant et en semaine après la date de la fête. La variable semaine de collecte introduite dans l'analyse a permis de distinguer la période ordinaire, la semaine avant la fête et la semaine après la fête. L'Anova du programme Spss a été utilisé pour déterminer l'importance des effets des différents facteurs qualitatifs. Un test $\mathrm{t}$ de Student a servi à comparer deux à deux les prix de mouton dans les différentes classes des facteurs.

\section{RESULTATS}

Le marché étant ouvert, certains animaux ont échappé au contrôle des enquêteurs. Par ailleurs, le client ne choisissait que parmi 
les moutons que lui offrait le marché. Par conséquent, son choix, modulé par la disponibilité des animaux sur le marché, a influencé les données obtenues. Les effectifs d'animaux enregistrés ne sont pas absolus pour la période de l'étude.

\section{Animaux commercialisés}

Les mesures ont porté sur 552 moutons parmi ceux qui ont été commercialisés sur le marché. Certains clients ont refusé que leurs animaux subissent des mensurations; le nombre d'animaux observés représentait 18 p. 100 des 3060 moutons sortis par l'accès au marché contrôlé par les enquêteurs. Le nombre de moutons enquêtés durant la semaine précédant chacune des fêtes a été plus important que celui des moutons enquêtés après la fête. Ce phénomène a été plus net après la fête de la Tabaski qu'après les autres fêtes (tableau I). De même, il a été observé plus d'ovins vendus en périodes festives qu'en périodes non festives.

\section{Effet des facteurs morphologiques sur le prix des animaux}

L'ACP normée a montré une forte corrélation entre le prix du mouton et les paramètres morphologiques. Toute l'information de l'ACP a été résumée dans le premier axe où se trouvaient 72,5 p. 100 de l'inertie totale. Tous les paramètres corporels intrinsèques ont été corrélés entre eux et avec le prix de vente du mouton. Ce prix a été fortement corrélé avec le poids vif $(\mathrm{r}=0,87)$ (tableau II). Il a augmenté régulièrement avec le poids vif jusqu'à $44 \mathrm{~kg}$. Au-delà, son accroissement est devenu irrégulier (figure 1). Les facteurs corporels ont fortement influencé le prix de vente des moutons. Pendant la Tabaski, en dehors du poids vif, c'est la hauteur au garrot qui a été la plus corrélée avec le prix $(r=0,70)$ alors

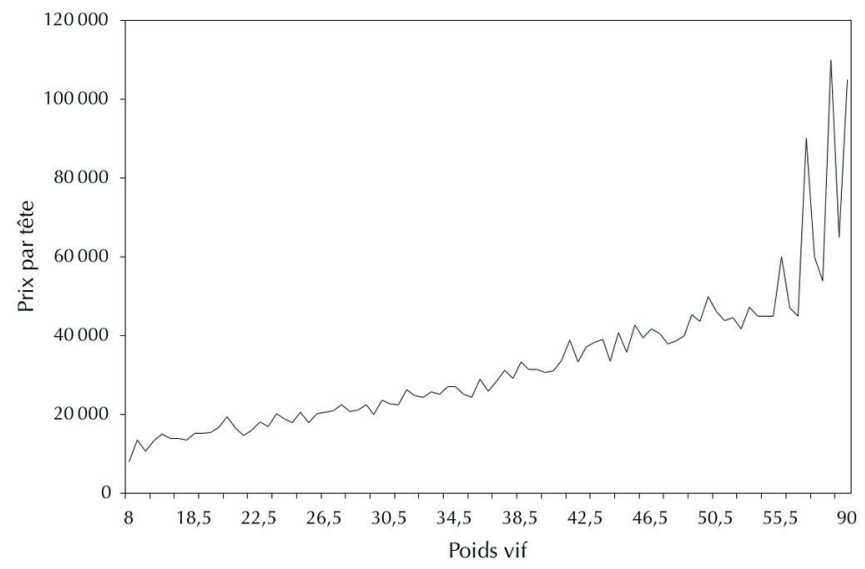

Figure 1 : prix moyen (Fcfa) du mouton en fonction de son poids vif $(\mathrm{kg})$.

que pour les autres périodes de l'année, c'est le périmètre thoracique qui a été le plus corrélé avec le prix après le poids vif.

L'analyse de régression linéaire a montré une influence significative $(\mathrm{P}<0,05)$ des variables poids vif, fête et longueur de la corne sur le prix de l'animal. Le modèle utilisé a permis d'expliquer 81,7 p. 100 de la variation totale du prix payé par animal. Cette analyse de régression a montré que lorsque le poids vif augmentait d'un kilogramme, le prix de vente augmentait de 924 Fcfa. En éliminant le poids vif du modèle de régression, la hauteur au garrot et la longueur des cornes ont déterminé le prix de l'animal. Dans ce cas, le modèle ne permettait d'expliquer que 54 p. 100 de la variation totale du prix.

\section{Tableau I}

Nombre de moutons comptés durant les deux semaines d'enquête autour des fêtes sur le marché de Bouaké entre juillet 2001 et juin 2002

\begin{tabular}{lcccc} 
Période d'observation & Tabaski & Fin Ramadan & Pâques & Noël \\
& Effectif (\%) & Effectif (\%) & Effectif (\%) & Effectif (\%) \\
\hline Semaine avant la fête & $798(92)$ & $378(85,5)$ & $229(60)$ & $300(63)$ \\
Semaine après la fête & $68(8)$ & $64(14,5)$ & $152(40)$ & $176(37)$ \\
Total des deux semaines & $867(100)$ & $442(100)$ & $381(100)$ & $476(100)$
\end{tabular}

\section{Tableau II}

Matrice de corrélations des différentes variables

\begin{tabular}{|c|c|c|c|c|c|c|c|}
\hline & Prix & Poids vif & $\begin{array}{l}\text { Hauteur } \\
\text { au garrot }\end{array}$ & $\begin{array}{l}\text { Périmètre } \\
\text { thoracique }\end{array}$ & $\begin{array}{l}\text { Longueur } \\
\text { queue }\end{array}$ & $\begin{array}{l}\text { Longueur } \\
\text { cou }\end{array}$ & $\begin{array}{c}\text { Longueur } \\
\text { oreille }\end{array}$ \\
\hline Prix & 1 & & & & & & \\
\hline Poids vif & 0,876 & 1 & & & & & \\
\hline Hauteur au garrot & 0,758 & 0,878 & 1 & & & & \\
\hline Périmètre thoracique & 0,656 & 0,743 & 0,718 & 1 & & & \\
\hline Longueur queue & 0,620 & 0,718 & 0,772 & 0,619 & 1 & & \\
\hline Longueur cou & 0,645 & 0,697 & 0,664 & 0,543 & 0,613 & 1 & \\
\hline Longueur oreille & 0,571 & 0,648 & 0,681 & 0,486 & 0,691 & 0,589 & 1 \\
\hline
\end{tabular}


La longueur moyenne des cornes à tout âge et toutes races confondues a été de $16,3 \mathrm{~cm}$ avec des extrêmes de $6 \mathrm{~cm}$ et $40 \mathrm{~cm}$. La majorité des animaux à longues cornes $(90$ p. 100) étaient des Sahéliens. Les Djallonké ont détenu la plus forte proportion d'animaux à petites cornes (62 contre 28,8 p. 100 pour les Sahéliens et 8,8 p. 100 pour les métis).

\section{Variations du prix des moutons pendant les périodes des fêtes}

A l'exception de la fête de Pâques, les prix des moutons vifs ont été légèrement plus élevés dans la semaine précédant la fête que durant celle qui a suivi les fêtes. Mais les différences entre les prix d'avant et d'après les fêtes n'ont pas été significatives $(\mathrm{P}>0,05)$ (tableau III). De même, les poids vifs d'avant et d'après les fêtes des moutons ont été identiques $(\mathrm{P}>0,05)$. La hauteur au garrot et le périmètre thoracique d'avant et d'après les fêtes, pris isolément, ont été identiques (tableau IV). Au cours de la semaine précédant les fêtes, des différences significatives $(\mathrm{P}<0,05)$ de prix de mouton et du kilogramme de poids vif ont été notées entre les fêtes. Les prix du mouton vif et du kilogramme de poids vif ont été plus élevés à la Tabaski qu'à Noël, Pâques et en fin Ramadan. Ces prix ont été plus faibles à Noël. L'accroissement le plus élevé du prix du kilogramme a été enregistré en période de la Tabaski. En effet, l'augmentation d'un kilogramme du poids du mouton faisait crô̂tre les prix de 1114 Fcfa à la Tabaski, mais seulement de 584 Fcfa à Noël, 894 Fcfa à Pâques et 732 Fcfa en fin Ramadan.

Par ailleurs, les prix du mouton et du kilogramme vif calculé ont été plus élevés $(\mathrm{P}<0,05)$ pour les Sahéliens que pour les Djallonké et les métis qui ont été vendus à des prix de kilogramme vif identiques $(\mathrm{P}>0,05)$.

\section{Tableau III}

Comparaison du poids vif ( $\mathrm{kg}$ ) et des prix (Fcfa) avant et après les fêtes sur le marché de Bouaké entre juillet 2001 et juin 2002

\begin{tabular}{|c|c|c|c|}
\hline $\begin{array}{l}\text { Période } \\
\text { d'observation }\end{array}$ & Paramètre & $\begin{array}{c}\text { Semaine } \\
\text { avant la fête }\end{array}$ & $\begin{array}{c}\text { Semaine } \\
\text { après la fête }\end{array}$ \\
\hline \multirow[t]{3}{*}{ Tabaski } & Poids vif & $34,08^{a}$ & 30,82 a \\
\hline & Prix/mouton & $36424^{a}$ & $36000^{a}$ \\
\hline & Prix/kg vif & $918,77^{a}$ & $924,86^{a}$ \\
\hline \multirow[t]{3}{*}{ Fin Ramadan } & Poids vif & $33,75^{a}$ & $31,30^{\mathrm{a}}$ \\
\hline & Prix/mouton & $25517^{a}$ & $24600^{a}$ \\
\hline & Prix/kg vif & $751,08^{a}$ & $802,10^{a}$ \\
\hline \multirow[t]{3}{*}{ Pâques } & Poids vif & $36,14^{\mathrm{a}}$ & $35,65^{\mathrm{a}}$ \\
\hline & Prix/mouton & $28468^{a}$ & $31859^{a}$ \\
\hline & Prix/kg vif & $799,81^{a}$ & $894,44^{b}$ \\
\hline \multirow[t]{3}{*}{ Noël } & Poids vif & $34,08^{a}$ & $30,82^{a}$ \\
\hline & Prix/mouton & $25615^{a}$ & $22286^{b}$ \\
\hline & Prix/kg vif & $745,10^{a}$ & $718,14^{a}$ \\
\hline \multirow{3}{*}{$\begin{array}{l}\text { Période ordinaire } \\
\text { de l'année }{ }^{*}\end{array}$} & Poids vif & \multicolumn{2}{|c|}{30,07} \\
\hline & Prix/mouton & \multicolumn{2}{|c|}{24006} \\
\hline & Prix/kg vif & \multicolumn{2}{|c|}{782} \\
\hline
\end{tabular}

\section{Tableau IV}

Hauteur au garrot $(\mathrm{cm})$ et périmètre thoracique $(\mathrm{cm})$ des moutons observés avant et après les fêtes sur le marché de Bouaké entre juillet 2001 et juin 2002

\begin{tabular}{|c|c|c|c|}
\hline $\begin{array}{l}\text { Période } \\
\text { d'observation }\end{array}$ & $\begin{array}{l}\text { Paramètre } \\
\text { mesuré }\end{array}$ & $\begin{array}{c}\text { Semaine } \\
\text { avant la fête }\end{array}$ & $\begin{array}{l}\text { Semaine } \\
\text { après la fête }\end{array}$ \\
\hline \multirow[t]{2}{*}{ Noël } & Hauteur au garrot & $73,43^{a}$ & $70,68^{a}$ \\
\hline & Périmètre thoracique & $75,74^{\text {a }}$ & $73,20^{a}$ \\
\hline \multirow[t]{2}{*}{ Pâques } & Hauteur au garrot & $73,85^{a}$ & $75,12^{a}$ \\
\hline & Périmètre thoracique & $77,62^{\text {a }}$ & $78,06^{\mathrm{a}}$ \\
\hline \multirow[t]{2}{*}{ Fin Ramadan } & Hauteur au garrot & $75,17^{\text {a }}$ & $68,50^{b}$ \\
\hline & Périmètre thoracique & $77,96^{\mathrm{a}}$ & $73,05^{\mathrm{a}}$ \\
\hline \multirow[t]{2}{*}{ Tabaski } & Hauteur au garrot & $77,50^{a}$ & $76,71^{a}$ \\
\hline & Périmètre thoracique & $77,75^{\mathrm{a}}$ & $80,28^{a}$ \\
\hline
\end{tabular}

${ }^{\mathrm{a}, \mathrm{b}}$ Les moyennes suivies d'une même lettre sur la même ligne ne sont pas différentes $(\mathrm{P}<0,05)$ selon le test $\mathrm{T}$ de Student

Durant la semaine suivant les fêtes, les prix du mouton vif et du kilogramme vif ont été plus faibles $(\mathrm{P}<0,05)$ à la fête de Noël qu'aux fêtes de la Tabaski et de Pâques. Des différences significatives $(\mathrm{P}<0,05)$ de poids vif et de prix ont été observées entre les trois types génétiques de moutons. Les prix des moutons Sahéliens ont été plus élevés que ceux des moutons Djallonké et des métis qui ont été vendus à des prix identiques. Cependant, le prix du kilogramme vif calculé a été identique pour les trois types génétiques.

Une fois le poids vif éliminé du modèle de régression, les principaux facteurs de variation significatifs $(\mathrm{P}<0,05)$ ont été la hauteur au garrot la semaine avant la fête et le périmètre thoracique la semaine après la fête.

\section{Variations du prix des moutons au cours des périodes ordinaires}

Des différences très significatives $(\mathrm{P}<0,001)$ de poids vif et de prix de mouton ont été observées entre les types génétiques, tandis que le prix du kilogramme de poids vif a été identique $(\mathrm{P}<0,05)$. Les moutons Sahéliens et métis ont coûté plus cher que les moutons Djallonké (tableau V). Par ailleurs, le prix du mouton a été plus élevé en février qu'en juillet, août et septembre (respectivement 27887 Fcfa contre 22136 et 20711 Fcfa).

\section{Tableau V}

Prix du mouton (Fcfa) durant la période ordinaire sur le marché de Bouaké entre juillet 2001 et juin 2002 par type génétique

\begin{tabular}{|lrrr|}
\hline Paramètre observé & Djallonké & Sahélien & \multicolumn{1}{c}{ Métis } \\
\hline Prix/mouton (Fcfa) & $18526^{\mathrm{a}}$ & $27376^{\mathrm{b}}$ & $25444^{\mathrm{b}}$ \\
Prix/kg vif (Fcfa) & $790^{\mathrm{a}}$ & $771^{\mathrm{a}}$ & $810^{\mathrm{a}}$ \\
\hline
\end{tabular}

${ }^{\mathrm{a}, \mathrm{b}}$ Les moyennes suivies d'une même lettre sur la même ligne ne sont pas différentes $(\mathrm{P}<0,05)$ selon le test $\mathrm{T}$ de Student 


\section{DISCUSSION}

Le poids vif a été un déterminant constant du prix du mouton à toutes les périodes de l'année. Son influence sur le prix des moutons a été déjà montrée $(8,19)$. L'effet prépondérant du poids vif sur le prix des moutons, observé aussi à Dakar (12), a confirmé le rôle fondamental du poids dans la détermination du prix du mouton à la Tabaski (établi par tête de mouton). Mais le poids vif n'a pas été le seul déterminant du prix parmi les facteurs morphologiques (1). En effet, la longueur des cornes, positivement corrélée au poids vif, a significativement influencé les prix de l'animal vif et du kilogramme de poids vif.

Pendant les fêtes musulmanes et particulièrement à la Tabaski, les transactions commerciales ont concerné surtout les moutons Sahéliens qui possédaient une hauteur au garrot élevée et de longues cornes. En plus du poids vif, la taille des animaux a joué un rôle capital dans le choix des clients pour la Tabaski. Juste avant la Tabaski, le prix des béliers augmentait $(1,9,10)$, surtout les plus grands et les mieux conformés tels que les Sahéliens (5) dont le prix au kilogramme vif observé a été plus élevé que celui des Djallonké et des métis. Ceci peut très bien se comprendre dans la mesure où, pour le sacrifice religieux de la Tabaski, les musulmans qui sont nombreux dans la ville ont une préférence prononcée pour les moutons Sahéliens. Par ailleurs, contrairement aux moutons Djallonké et métis, la presque totalité (99 p. 100) des moutons Sahéliens commercialisés sur le marché de Bouaké ont été importés. Ainsi, le besoin prononcé pour les moutons Sahéliens qui sont presque tous importés expliquerait la cherté de ces moutons pendant la Tabaski et même en dehors de cette fête.

Les prix des moutons avant et après les fêtes ont été identiques dans cette étude, contrairement aux résultats d'Abba (1). Le poids vif a été le principal facteur qui a déterminé le prix du mouton en toute période d'observation. En le retirant du modèle de régression, la hauteur au garrot et le périmètre thoracique sont devenus les principaux déterminants significatifs du prix, respectivement la semaine avant et la semaine après les fêtes. Ces deux paramètres (avant et après la fête) pris individuellement étant identiques, les prix qu'ils déterminent ne peuvent être qu'identiques. Cependant, la différence de prix du kilogramme vif observée entre la semaine avant et la semaine après Pâques serait due probablement à une défaillance de la fixation des prix par les vendeurs car les poids vifs avant et après Pâques étaient identiques $(\mathrm{P}<0,05)$.

Certains producteurs ont très bien compris ces variations de prix du mouton selon le type génétique et les fêtes. Au Sénégal, la demande en moutons pour la Tabaski est particulièrement élevée à Dakar où la population est à 94 p. 100 musulmane (13). Ainsi, en prévision de la période de la Tabaski, ils pratiquent de l'embouche ovine (6) afin de mettre sur le marché de gros animaux. Cette embouche de moutons est souvent organisée par les femmes près des habitations. Les produits obtenus sont surtout commercialisés lors des fêtes religieuses musulmanes et chrétiennes (4). L'implantation de l'islam a été un facteur non négligeable pour le développement de l'élevage ovin au Cameroun (18) où, à Maroua, il contribue largement à la satisfaction des besoins des populations musulmanes lors des fêtes religieuses (16).

Les transactions commerciales sur le marché ont montré que le poids vif a été un facteur primordial dans la fixation du prix du mouton bien que les animaux n'aient jamais été pesés à cet effet. De même, le prix du kilogramme vif calculé a varié pratiquement dans le même sens que celui du poids vif. Ce prix du kilogramme vif a été identique entre les types génétiques et à l'intérieur de chaque période d'observation. Cela montre bien qu'il existe une constance appréciable dans la fixation du prix du mouton par ces commerçants. Ainsi, cette détermination du prix du mouton de manière subjective révèle que les commerçants ont acquis une expérience solide. Leur efficacité dans la détermination subjective des prix du mouton sur le marché est comparable à celle de la technique moderne basée sur la pesée des animaux. Par ailleurs, les propriétaires des troupeaux de moutons possèdent aussi une certaine expérience dans la fixation des prix des animaux.

En effet, le prix du mouton vendu en période ordinaire (24 006 Fcfa) est comparable au prix pratiqué pour les ventes à domicile par les propriétaires eux-mêmes qui s'élève à 22600 Fcfa en moyenne (17). Cette expertise semble être partagée par les principaux acteurs de la filière ovine urbaine.

Le prix de vente du mouton Djallonké (18 526 Fcfa) observé sur le marché a été plus élevé que celui qui a été enregistré lors des transactions commerciales en 2001 au sein de l'abattoir des petits ruminants et qui était de $16890 \mathrm{Fcfa}$ en moyenne pour un poids identique (Touré, non publié). Le Djallonké était le type génétique le plus commercialisé à l'abattoir des petits ruminants. Cette différence de prix était due à la spéculation entretenue par les intermédiaires, d'une part, et, d'autre part, à une contrainte de temps éprouvée par les paysans qui ravitaillaient l'abattoir.

Une fois à l'abattoir avec leurs animaux, les paysans tenaient à les vendre le même jour et à retourner chez eux. Conscients de cette contrainte de temps, les clients ou intermédiaires faisaient chuter les prix à leur avantage. Cette pratique se rencontre aussi au Cameroun où les intermédiaires interceptent sur les voies d'entrée du marché les vendeurs pressés ou venant des localités éloignées, qui doivent vite se défaire de leur marchandise pour regagner leur domicile ou éviter de payer la taxe (10). Cette pratique dans le commerce du mouton est également signalée au Sénégal (9). Ainsi, outre les critères de choix des clients, la spéculation à expression variable semble prépondérante dans le mécanisme de fixation du prix du mouton. Les prix des moutons n'étaient pas seulement élevés durant les périodes des fêtes, car, en période ordinaire, ils variaient selon les mois, révélant ainsi l'influence de la date d'achat du mouton $(1,14,15,19)$.

Les fêtes, en particulier la Tabaski, restent le moment de meilleure valorisation commerciale des moutons. La production intensive et massive doit viser ces périodes de commercialisation optimale. Par conséquent, la production des éleveurs doit tenir compte du format et de la période de commercialisation. Pour les fêtes musulmanes, la production doit s'orienter vers des animaux de grande taille, à savoir les Sahéliens et les métis. Pour les autres périodes, festives ou non, toutes catégories d'animaux peuvent être produits, car leur achat est soumis à des besoins individuels spécifiques. Ces besoins étant très variables (consommation domestique simple, mariage, dot, baptême, sacrifice) et éparpillés sur l'année, toute catégorie de mouton peut faire l'objet de transaction commerciale.

Ainsi, à l'aide de ces informations commerciales locales, les éleveurs de moutons peuvent mieux programmer la reproduction des animaux afin d'obtenir des produits commercialisables aux périodes où les prix sont les plus rémunérateurs sur le marché. Ils peuvent, ainsi, mieux valoriser leur production. Par ailleurs, les importateurs de moutons pourront cibler aussi ces périodes favorables du marché.

\section{CONCLUSION}

La technique de fixation de prix des moutons sur le marché est une méthode endogène efficace qui met en évidence l'influence du format des animaux, des périodes festives et des mois au cours de l'année. Les fêtes, en particulier la Tabaski, restent le moment 
de meilleure valorisation commerciale des moutons. Cette dynamique du prix des moutons peut être utilisée par les producteurs et les importateurs comme base de données commerciales utiles à la programmation de vente aux moments propices. L'exploitation des variations de ces transactions commerciales du mouton sur le marché par les opérateurs économiques devrait tenir compte de l'existence des autres sites de commercialisation dans la ville qui ne partagent pas forcément la même dynamique de prix.

\section{BIBLIOGRAPHIE}

1. ABBA D., 1998. Variation du prix du bélier dans la zone sahélienne du Cameroun. Tropicultura, 16-17:15-17.

2. AMEGEE Y., 1983. Le mouton de Vogan (croisé Djallonké x Sahélien au Togo. Revue Elev. Méd. vét. Pays trop., 36 : 79-84.

3. BARRY M.B., 1985. Le rôle des petits ruminants dans la stratégie d'intensification des systèmes de production en Côte d'Ivoire. In : Wilson R.T., Bourzat D. (eds), Les petits ruminants dans I'agriculture africaine. Addis Abeba, Ethiopie, Cipea, p. 199-206.

4. BULDGEN A., DETIMMERMAN F., PIRAUX M., COMPERE R., 1992 Les techniques d'embouche de moutons en région soudano-sahélienne sénégalaise. Revue Elev. Méd. vét. Pays trop., 45 : 321-328.

5. CENTRES J.M., 1996. L'élevage et l'agriculture en zones urbaine et périurbaine dans deux villes sahéliennes: Bamako et Bobo-Dioulasso. Cah. Agric., 5 : 373-381.

6. CHEVASSUS N., 1992. Enquête technico-économique sur l'embouche ovine en période de Tabaski à Garoua (Nord Cameroun). Maisons-Alfort, France, Cirad-emvt, 107 p.

7. DEVENDRA C., 1981. Potential of sheep and goats in less developed countries. J. Anim. Sci., 51: 461-473.

8. EHUI S.K., SHAPIRO B.I., YAPI-GNAORE V.C., 1995. Peri-urban livestock production and development in sub-Saharan africa: a review of the constraints and opportunities. In: Proc. 8th International conference AITVM, Livestock production and diseases in the Tropics, Livestock production and human welfare, Berlin, Germany. Utrecht, the Netherlands, AITVM, p. 151-163.
9. FAuGere O., DOCKES A.C., PERROT C., FAuGere B., 1990. L'élevage traditionnel des petits ruminants au Sénégal. II. Pratiques de conduite et d'exploitation des animaux chez les éleveurs de la région de Louga. Revue Elev. Méd. vét. Pays trop., 43 : 261-273.

10. KILANGA S., HECHEKET D.A., NGAMBIA F.R., 1999. L'élevage ovin périurbain à Maroua dans l'extrême nord du Cameroun. In : Moustier P., Mbaye A., De Bon H., Guerin H., Pages J., éds, Actes de l'atelier Agriculture périurbaine en Afrique subsaharienne, Montpellier, France, 20-24 avril 1998. Montpellier, France, Cirad, 278 p. (Coll. Colloques)

11. LANDAIS E., BASSEWITZ H., 1982. Détermination de l'âge des moutons Djallonké du Nord de la Côte d'lvoire par examen de leur dentition. Revue Elev. Méd. vét. Pays trop., 35 : 57-62.

12. LY C., 1997. Le mouton de Tabaski à Dakar. Facteurs explicatifs du prix de vente. Tropicultura, $15:$ 105-108.

13. MISSOHOU A., LY C., DIEDHIOU M., TILLARD E., DIOUF S., 1995. Elevage citadin de moutons à Dakar. In : Proc. 8th International conference AITVM, Livestock production and diseases in the Tropics, Livestock production and human welfare, Berlin, Germany. Utrecht, the Netherlands, AITVM, $173 \mathrm{p}$.

14. MOUSSA C., NIO A, N'GO TAMA A.C, CARDINALE E, SALI B, 1997. Formation des prix au producteur et impact de la dévaluation du franc CFA sur les stratégies de production des petits ruminants. Montpellier, France, Cirad, p. 514 (Coll. Colloques)

15. OUEDRAOGO M., 1996. Les perspectives de développement de l'élevage des petits ruminants au Togo: cas de la région des savanes. Mémoire DESS-PARC, université de Montpellier II, Cirad, France, 86 p.

16. THYS E., EKEMBE T., 1992. Elevage citadin des petits ruminants à Maroua (province de l'Extrême Nord du Cameroun). Cah. Agric., 1 : 249-255

17. TOURE G., OUATTARA Z., 2001. Elevage urbain des ovins par les femmes à Bouaké, Côte d'Ivoire. Cah. Agric., 10 : 45-49.

18. VALLERAND F., BRANCKAERT R., 1975. La race ovine Djallonké au Cameroun. Potentialités zootechniques, conditions d'élevage, avenir. Revue Elev. Méd. vét. Pays trop., 28 : 523-545.

19. YAPI-GNAORE C.V., ZANA O., TOURE G., 1994. Evolution des prix des moutons autour de la fête de Tabaski sur le marché à petits ruminants de Bouaké. In : Socioéconomie de l'élevage ovin en milieu peri-urbain. Rapport semestriel d'activités. Bouaké, Côte d'Ivoire, Idessa, 22 p.

Reçu le 08.10.2003, accepté le 12.09.2005 


\section{Summary}

Toure G., Ouattara Z., Yapy-Gnaore V., Yo T., Tanoh K.G. Sheep Commercialization in Bouake, Côte d'Ivoire: Price Variations at the Small Ruminants Market

A survey based on a one-time questionnaire for sheep customers was conducted at the small ruminants market of Bouake. The aim was to determine the dynamics of sheep price during the year. In the data collected, which concerned animal price and some of their physical characteristics, a particular attention was given to the periods of major holidays. A total of 552 sheep commercialized at the market were included in the survey. The number of sheep sold a week before a holiday was higher than that of sheep sold after the holiday. The small ruminants market of Bouake was mainly supplied with imported sheep $(81 \%)$ with a majority of the Sahelian type $(60 \%)$. The traditional technique of defining sheep retail price with visual appraisal and without any measuring instrument provided similar results to those obtained when setting the price based on the exact weight. The traders proved to be quite experienced in that appraising activity. Live weights and holiday periods significantly affected the sheep price. Large sheep, mainly of Sahelian type, were specially sold over Muslim holidays. In the weeks preceding the holidays, the highest sheep price recorded was for the Tabaski (on average 39,705 CFAF per Sahelian sheep and 26,750 CFAF per Djallonke). The lowest sheep price was recorded for the Sahelian sheep at Christmas time $(28,105$ CFAF/head) and for the Djallonke sheep over New Year's $(17,937$ CFAF/head). The sheep price was mainly related to animal weights and holiday periods throughout the year, which modulated the customer's choice.

Keywords: Sheep - Marketing - Retail marketing - Price Côte d'Ivoire.

\section{Resumen}

Toure G., Ouattara Z., Yapy-Gnaore V., Yo T., Tanoh K.G. Comercialización de corderos en Bouaké, Costa de Marfil: variaciones de precios en el mercado de los pequeños rumiantes

Se llevó a cabo una encuesta, mediante un cuestionario único, entre los clientes de corderos del mercado de ganado de Bouaké. El objetivo fue la determinación del comportamiento del precio del cordero durante el año. La recolección de datos, con una atención particular a los periodos de grandes fiestas anuales, se centró en el precio y algunas características físicas de los animales. La encuesta concernió 552 corderos comercializados en el mercado. La cantidad de corderos vendidos durante la semana anterior a cada una de las fiestas fue más elevada que después de cada fiesta. El mercado de los pequeños rumiantes de Bouaké es sobretodo abastecido de corderos importados (81\%), en su gran mayoría de tipo Sahelino $(60 \%)$. La forma tradicional de fijar el precio de los corderos mediante una apreciación visual de la corpulencia, sin instrumentos de medida, a dado resultados comparables a la determinación del precio mediante pesas. Los vendedores han demostrado una buena experiencia en esta actividad. El peso vivo y los periodos de fiesta han influenciado significativamente el precio del cordero. Los corderos de gran tamaño, en su mayoría de tipo Sahelino, se vendieron principalemente durante los periodos de fiestas musulmanas. Durante las semanas que precedieron las fiestas, el precio del cordero más elevado se registró en Tabaski (en promedio 39 705 Fcfa/cordero Sahelino y 26750 Fcfa/cordero Djallonké); el precio del cordero más bajo se obtuvo en Navidad para el cordero Sahelino (28 $105 \mathrm{Fcfa} / \mathrm{cabeza}$ ) y en Año nuevo para el cordero Djallonké (17 $937 \mathrm{Fcfa} / \mathrm{cabeza}$ ). El precio de los cordero estuvo principalmente ligado al peso de los animales y a los periodos de fiestas anuales, los cuáles modularon la escogencia del cliente.

Palabras-clave: Ovino - Mercadeo - Venta al por menor Precio - Côte d'Ivoire. 